\title{
Design and Optimization of Lifting Mechanism in Hemming Machine Based on ADAMS
}

\author{
Juguang LIN $^{1,}$ a , Zhiwen JIANG ${ }^{1, b}$, Wenjie WU ${ }^{1}$, Lei CAI ${ }^{1}$ \\ ${ }^{1}$ Department of Mechanical and Automobile Engineering, Hefei University of Technology, Hefei, \\ 20009, China \\ aemail: 18255121315@163.com, bemail:893318083@qq.com
}

Keywords: Hemming Machine; Lifting Mechanism; Virtual Prototype

\begin{abstract}
Hemming machine is an important method of craft of the hemming of automobile panels. According to the working characteristic of hemming machine, designing a kind of lifting mechanism of great reinforcement ratio that moves steadily. Establishing virtual prototype model of lifting mechanism with dynamic simulation software ADAMS, with such two factors stationarity and reinforcement ratio as indicators, having a comparative choice of the driving method of hemming machine, through the sensitivity analysis of the pressure of hinged rod by studying singular length of rod, finding out the appropriate designing variable, to which adding constraint condition and treating the average stress in the place where the rod hinges in a singular trip as the objective function, then having the minimum value optimization so as to get the optimum parameter of rod.
\end{abstract}

\section{Introduction}

To hem closures, several technologies are available on the market, such as die hemming, tabletop hemming, Robot roller hemming [1][2] and Hemming machine. Compared with the other three ways, the Hemming machine has the advantages of small size, high efficiency and low equipment cost. Although its flexibility is worse than Robot roller hemming, but the quality and the stability of hemming are far better than the Robot roller hemming, so it is widely used in automobile manufacturers at home and abroad [3].

Figure 1 illustrates the three-dimensional structure of Hemming machine, its mechanical structure is mainly composed of base, lifting mechanism, caul, sliding guide rail, hemming mechanism and its positioning clamping mechanism, etc.

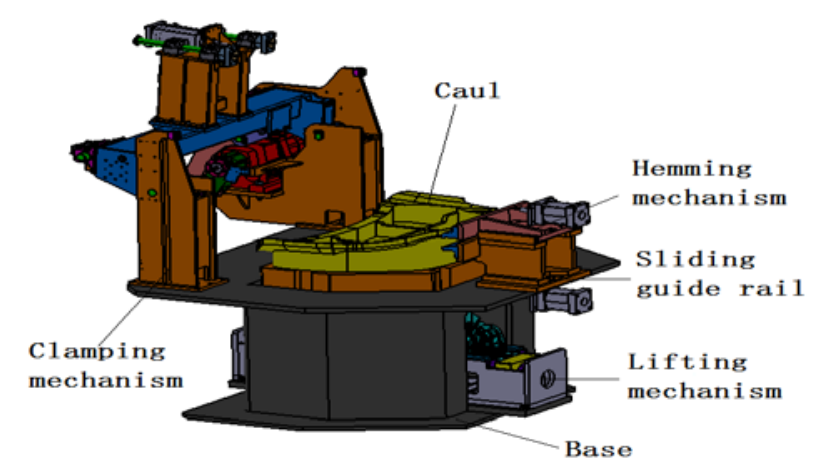

Fig.1. The three-dimensional structure of Hemming machine

\section{Design of lifting mechanism}

Lifting mechanism in a hemming process requires repeated lifting many times, which requires lifting mechanism has good movement stability, and because hemming will produce a lot of pressure [4], lifting mechanism should has great reinforcement ratio. In order to meet the job requirements, we designed a screw-toggle tandem transmission mechanism. As shown in Figure 2 (a), when the mechanism rising, the screw is turned for the first driven. Then screw drive nut doing 
linear motion, urging force is transmitted to the rod, so that the triangle toggle mechanism is rotated, the pressure angle decreases, energizing the second booster rod, drag the base complete lifting action. This mechanism's working stroke is large and adjustable, with good low speed characteristic and large reinforcement ratio. Screw-toggle tandem transmission mechanism is completely symmetrical, take half a simplified model shown in Figure 2 (b).

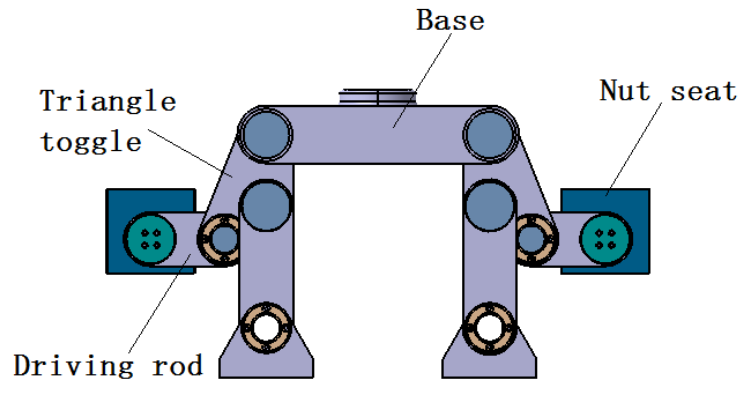

(a) Lifting mechanism model

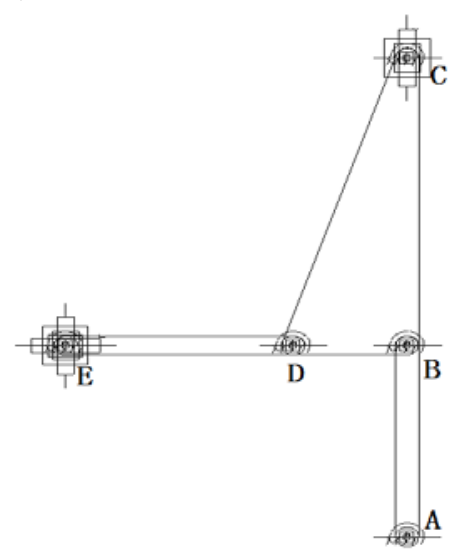

(b) Lifting mechanism simplified model

Fig.2. The model

In the virtual prototype model, the initial coordinates of each point as shown in Table 1.

Table 1 Initial coordinates of each point

\begin{tabular}{cccccc}
\hline Point & A & B & C & D & E \\
\hline $\mathrm{X}$ & 0 & 0 & 0 & -100 & -300 \\
\hline $\mathrm{Y}$ & 0 & 200 & 500 & 200 & 200 \\
\hline
\end{tabular}

The screw drive nut in two ways, the horizontal drive and vertical drive. Hemming work demand of lifting the largest height is $150 \mathrm{~mm}$. Under the same conditions, compare the two drives in output speed and reinforcement ratio in a rising travel, then we can get speed - stroke curve and pressure stroke curve, as shown in Figure 3.

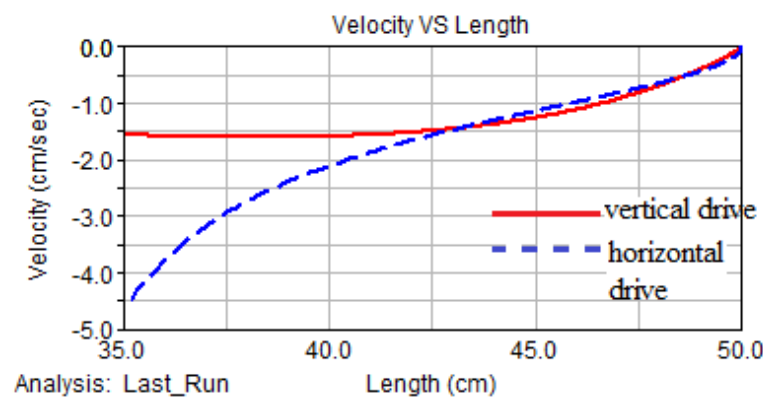

(a) Speed - stroke curve

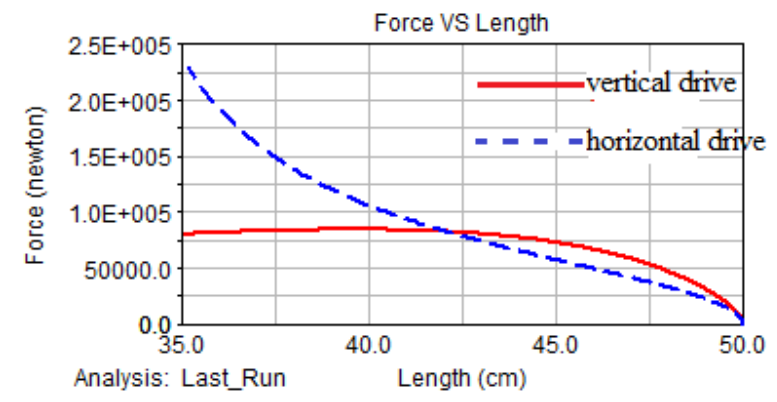

(b) Pressure - stroke curve

Fig.3. Vertical drive and horizontal drive motions features comparison

Analysis Figure 3 (a) shows, under the same driving conditions in the same stroke, the vertical drive, the rise of speed and acceleration is lower than the horizontal drive, more stable movement, help to improve the molding accuracy hemming. Figure 3 (b) shows when the load is $50000 \mathrm{~N}$, screw axial force suffered, namely the driving force needed to mechanism. It can be seen from the figure, the same load in curve within the period of $350 \mathrm{~mm}$ to $400 \mathrm{~mm}$, vertical driving force required to drive smaller, better performance of its booster. Between $400 \mathrm{~mm} \sim 500 \mathrm{~mm}$ curve, the output motion curves of two kinds of driving mode is close to. So choose the vertical drive.

\section{Optimization of lifting mechanism}

When hemming, a lot of pressure on the agency, in order to improve the performance of institutions [5], so that the hinge point of the force is reduced in the same load, to optimize the length of the rod. According to the design requirements, $A_{x}, A_{y}, B_{x}, C_{x}, C_{y}$ remains the same, will $B_{y}, D_{x}, D_{y} 、 E_{x} 、 E_{y}$ parameterized, get five design variables correspond to the following, 
DV_1: $B_{y}$ (B point ordinate),

DV_2: $D_{x}$ (D point abscissa),

DV_3: $D_{y}$ (D point ordinate),

DV_4: $E_{x}$ (E point abscissa),

DV_5: $E_{y}$ (E point ordinate)。

On the analysis of each variable separately, to study the sensitivity of the force on the hinges, variable scope for its standard value plus or minus $20 \%$, each variable within the scope of the change take 10 simulation values, to obtain the results shown in Table 2.

Table 2 The sensitivity of individual variables to the force rod

(1) Design variables

\begin{tabular}{cccccc}
\hline Variable & DV_1 & DV_2 & DV_3 & DV_4 & DV_5 \\
\hline Coordinate & $B_{y}$ & $D_{x}$ & $D_{y}$ & $E_{x}$ & $E_{y}$ \\
\hline Sensitivity & 97.4 & -171 & 199.8 & 64 & -59.4 \\
\hline
\end{tabular}

According to the sensitivity analysis, the sensitivity of DV_1, DV_2, DV_3 to the stress of the rod is larger, so it is set as design variables. The scope of each variable is,

(2) The objective function

$$
\begin{gathered}
100 \leq B_{y} \leq 200 ; \\
-100 \leq D_{x} \leq-60 ; \\
100 \leq D_{y} \leq 200 \text { 。 }
\end{gathered}
$$

In this paper, take the maximum force of hinge point $\mathrm{D}$ in a stroke within the average stress minimum as the optimization objective. Create its force measurement function, $F_{d}$ : MEA_F $F_{d}=f\left(B_{y}, D_{x}, D_{y}\right)$

(3) Constraints

Firstly, $\mathrm{C}$ point position measurement function is established, MEA $C_{y}$. The lifting mechanism stroke is $150 \mathrm{~mm}$, so the constraints $C$ position is $350 \leqslant \mathrm{MEA} C_{y} \leqslant 500$. Secondly, we must meet the conditions exist triangle toggle [6],

$$
\begin{aligned}
& \sqrt{B_{x}{ }^{2}+\left(B_{y}-C_{y}\right)^{2}}<\sqrt{D_{x}{ }^{2}+\left(C_{y}-D_{y}\right)^{2}}+\sqrt{\left(B_{x}-D_{y}\right)^{2}+\left(B_{y}-D_{y}\right)^{2}} \\
& \sqrt{D_{x}{ }^{2}+\left(C_{y}-D_{y}\right)^{2}}<\sqrt{\left(B_{x}-D_{x}\right)^{2}+\left(B_{y}-D_{y}\right)^{2}}+\sqrt{B_{x}{ }^{2}+\left(B_{y}-C_{y}\right)^{2}} \\
& \sqrt{\left(B_{x}-D_{x}\right)^{2}+\left(B_{y}-D_{y}\right)^{2}}<\sqrt{B_{x}{ }^{2}+\left(B_{y}-C_{y}\right)^{2}}+\sqrt{D_{x}{ }^{2}+\left(C_{y}-D_{y}\right)^{2}}
\end{aligned}
$$

Considering the practical factors, the gravity of the lever, inertial force, friction, etc is loaded into the model. Select OPTDES-GRG optimization algorithms, optimized results as shown in Table 3,

Table 3 The coordinates of each point before and after optimization

\begin{tabular}{cccc}
\hline Design variables & $B_{y} / \mathrm{mm}$ & $D_{x} / \mathrm{mm}$ & $D_{y} / \mathrm{mm}$ \\
\hline Before optimization & 200 & -100 & 200 \\
\hline After optimization & 180 & -63 & 113 \\
\hline
\end{tabular}

Simulate the virtual prototype again after optimization, then get the motion characteristic curve and compare with pre-optimized, as shown in Figure 4.

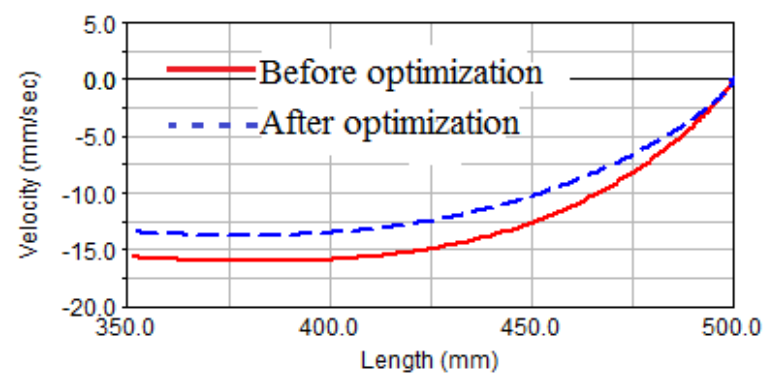

(a) Speed - stroke curve

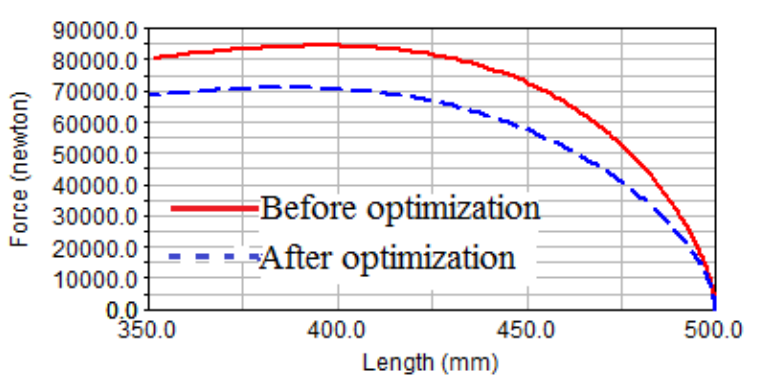

(b) Pressure - stroke curve

Fig.4. Motion characteristics comparison before and after optimization

The optimized output speed more smoothly, is helpful to improve the quality of the hemming precision. The original force $83355 \mathrm{~N}$ at D point, has reduced to $70644 \mathrm{~N}, 15.3 \%$ was optimized. 


\section{Conclusion}

The output characteristic of lifting mechanism directly affects the quality and efficiency of hemming. In reference to previous studies, based on the operating characteristics for hemming plane, and designed a screw - toggle tandem lifting mechanism. Optimized lifting mechanism more meet job requirements, and improve the quality and efficiency of hemming. Study on Simulation of Virtual Prototyping Based on ADAMS, can effectively optimize the structure parameters of the mechanism, to achieve the best design goals.

\section{References}

[1] Jonkers, B., Hemming Basics, an overview of all the different hemming technologies and their variants. Polynorm Automotive, unschoten, 2005. p. 22-26.

[2] P. Jimbert, I. Eguia, I. Perez, M.A. Gutierrez, and I. Hurtado (2010)

Analysis and Comparative Study of Factors Affecting Quality in the Hemming of 6016-T4AA Performed by Means of Electromagnetic Forming and Process Characterization, J. Mater. Process. Technol.,2011, 211(5), p 916-924

[3] G. Zhang, H. Hao, X. Wu, S.J. Hu, K. Harper, and W. Faitel, An Experimental Investigation of Curved Surface-Straight Edge Hemming, J. Manuf. Processes, 2000, 2(4), p 241-246

[4] J.E. Carsley, Microstructural Evolution During Bending: Conventionalvs. Roller Hemming, Trends in Materials and ManufacturingTechnologies for Transportation Industries, T.R. Bieler, J.E. Carsley,H.L. Fraser, J.W. Sears, and J.E. Smugeresky, Ed., TMS, 2005,p 169-174

[5] Zhu Jiuyou Robot roller hemming technology research [D] Hefei: Hefei University of Technology, 2010

[6] Nai Shunfeng Design of motion and force servo presses toggle mechanism Optimization[D] Guangdong: Guangdong University of Technology, 2012 